\title{
Ausaperung und Klimaverlauf am Beispiel des Griesgletschers
}

\section{Problemstellung}

Der Fragenkomplex Klima und Gletscher beschäftigte in den letzten Jahren nicht mehr nur Glaziologen und Klimatologen, sondern gewann durch Fragen der Veränderungen der Umwelt mit ihren Auswirkungen auf das Klima und durch Fragen der Nutzung der Hochgebirgsregionen auch für die allgemeine Geographie an Bedeutung.

Zwischen Gletscherschwankungen und Klimaverlauf bestehen enge Beziehungen. Der Massenhaushalt eines Gletschers wird ohne Verzögerung durch die herrschenden klimatischen Verhältnisse bestimmt: Die Mächtigkeit der Schneedecke (Akkumulation) und der Aufbrauch von Schnee, Firn und Eis (Ablation) ergeben die Massenbilanz. Fällt die Differenz zwischen Akkumulation und Ablation positiv aus, bewirkt dies eine Vergrößerung der Gletschermasse (positive Massenbilanz). Bei einer negativen Massenbilanz (Massenverlust) überwiegt die Ablation. Diese Massenhaushaltsänderungen übertragen sich mit einer gewissen Zeitverschiebung auf die Gletscherzunge und bewirken damit Vorstoß oder Rückzug.

Die Untersuchungen des vorliegenden Problemkreises mußten daher an einem ausgewählten Beispiel in zwei Richtungen geführt werden:

1. Verfolgen und festhalten des Ausaperungsverlaufes im Sommer.

2. Vergleich Massenhaushalt - Klimaverlauf mit Hilfe von Korrelationsrechnungen.

Das Pegelnetz, die 1ljährigen Massenhaushaltsbestimmungen durch die Abteilung für Hydrologie und Glaziologie der Versuchsanstalt für Wasserbau, Hydrologie und Glaziologie (VAW) an der ETH Zürich und die topographische und morphologische Einfachheit ließen den Griesgletscher (Obergoms) als für diese Untersuchungen geeignet erscheinen.

\section{Begriffe und Methode}

Die Massenbilanz (B) eines Gletschers wird am Ende eines Haushaltsjahres (30. Sept.) aus der Differenz der noch vorhandenen Altschneemenge (Akkumulation: C) und der sommerlichen Ablation (A) berechnet: $\mathrm{B}=\mathrm{C}-\mathrm{A}$
Zur Erfassung des Altschneevolumens dienten im Sommer 1972 auf dem Griesgletscher die ca. 40 Pegel der VAW. Die topographische Einfachheit der Gletscheroberfläche erlaubte die Extrapolation der Pegelwerte auf die entsprechende Höhenstufe und damit die Berechnung des gesamten Akkumulations- und Ablationsvolumens. Schon vorhandener Septemberneuschnee wird noch dem laufenden Haushaltsjahr zugeordnet.

Die International Commission of Snow and Ice der International Association of Scientific Hydrology (1969) definiert:

Akkumulation: Resultat aller Prozesse, die die Maße eines Gletschers vergrößern.

Ablation: Resultat aller Prozesse, die die Maße eines Gletschers verringern.

Unter Altschnee wird Schnee der Akkumulationsperiode des laufenden Haushaltsjahres verstanden, während mit Firn der Schnee aus früheren Haushaltsjahren bezeichnet wird.

Die Altschneelinie trennt Altschnee und Firn oder Eis, die Firnlinie Firn und Eis.

Der Wasserwert wird z. B. mit Hilfe eines Meßzylinders und einer Federwaage aus Probengewicht (kg)/Probengrundfläche $\left(\mathrm{m}^{2}\right)$ bestimmt. $1 \mathrm{~kg} / \mathrm{m}^{2}$ entspricht $1 \mathrm{~mm}$ Wasserhöhe.

\section{AusaperungsgesetzmäBigkeiten}

Mit zunehmender Erwärmung und Strahlung beginnt die Altschneelinie im Frühjahr höher zu steigen. Dabei kann beobachtet werden, daß sich Altschneelinie und Höhenkurven oftmals nicht decken. Das Höhersteigen der Altschneelinie verläuft nicht parallel zur Zunahme der Höhe. Manchmal ist eine klare Altschneelinie festzustellen, häufig löst sich jedoch die Altschneedecke in einzelne Flecken auf, die langsam verschwinden.

Beobachtet man in der gleichen Region den Ausaperungsproze $\beta$ über mehrere Jahre, so fallen die stets wiederkehrenden gleichen Konturlinien der Altschneedecke auf. Die einzelnen Aperfiguren treffen zwar, als Folge der klimatischen Unterschiede, nicht jedes Jahr zum gleichen Zeitpunkt ein, doch wiederholt sich in einer bestimmten Phase das gleiche Aperbild.

Martin Hasler, Gymnasiallehrer, Schönmattweg 22, 3123 Belp 
Auf diese Gesetzmäßigkeit stieß FRIEDEL (1952) bei Untersuchungen über die Niederschlagsverteilung im Hochgebirge. SLUPETZKY (1971) stellte dann fest, daß für die Konstanz der Aperfiguren die Faktoren Schneehöhe und Abschmelzenergie verantwortlich sind.

Die Schneehöhe ist eine Folge der Schneeablagerungen und -umlagerungen. Höhere Lagen erhalten mehr Schnee, ebenso sind Leeseiten bevorzugt. Die sich oft ändernden Winde bewirken während des Winters Schneeumlagerungen und damit eine Einnivellierung des Kleinreliefs. Muldenlagen weisen größere Schneemengen auf als Buckellagen. Die beiden Faktoren Höhe über Meer und das Kleinrelief bestimmen also die Schneehöhe.

Die Abschmelzenergie läßt sich aus der Wärmehaushaltsgleichung errechnen. Dabei überwiegt meistens der Anteil der Strahlung den Wärmestromanteil (HoINKES und WENDLER, 1968, HOEK, 1952). Einen unterschiedlichen Strahlungsanteil weisen die verschiedenen Expositionen auf: Gegen Süden geneigte Hänge sind gegenüber flachen Stellen, diese wiederum gegenüber Nordhängen bevorzugt. Die stärkere Trübung der Atmosphäre und die im Mittel stärkere Bewölkung in der zweiten Tageshälfte begünstigen zudem geringfügig Osthänge gegenüber Westhängen. Die Abnahme der Temperatur mit zunehmender Höhe bewirkt eine Verminderung des Wärmestroms und damit eine Abnahme der Abschmelzenergie mit zunehmender Höhe. Das Aperbild wird damit durch folgende Faktoren geprägt:

1. Niveauunterschiede

2. Kleinrelief

3. Exposition

Da alle 3 Faktoren in einem Untersuchungsgebiet konstant sind, werden jedes Jahr die gleichen Aperbilder durchlaufen. Die unterschiedlichen klimatischen Verhältnisse bestimmen nur den Ablauf, nicht jedoch das Bild der Ausaperung.

\section{Die Ausaperung des Griesgletschers im Sommer 1972}

Der für die Ausaperungsuntersuchungen ausgewählte Griesgletscher liegt am Ostende der südlichen Walliser Alpenkette in einer sich vom Bedrettotal ins obere Binntal erstreckenden Bündnerschieferzone. Der Gletscher besitzt eine einheitliche ENE-Exposition und zeichnet sich zudem als einfacher Zungengletscher durch eine niedrige Umrahmung aus. Die Gesamtlänge des Gletschers beträgt heute $5,4 \mathrm{~km}$. Er erreicht am Blinnenhorn ca. $3300 \mathrm{~m}$ und endet mit einem Abbruch am Stausee Gries auf $2386 \mathrm{~m}$.

Über den Wetterablauf im Sommer 1972 geben Fig. 1-3 Auskunft. Die Altschneegrenze erreichte nach einem niederschlagsarmen Winter Ende April ihren Normalstand. Ständige Neuschneefälle verzögerten im Mai den raschen Abbau der Schneedecke. Auch die Junitemperaturen lagen im Alpenraum um etwa $1^{\circ}$ unter dem Mittel. Erst in der zweiten Hälfte Juli und in der ersten Hälfte August kam es zu mehrtägigen Schönwetterperioden. Am 18/19. August wurde die Ausaperung durch einen Sommerneuschneefall unterbrochen und anfangs September durch weitere Schneefälle endgültig abgebrochen.

Für den Klimaverlauf im Sommer 1972 sind folgende Merkmale charakteristisch:

1. Das nur zögernde Höhersteigen der Altschneelinie im Frühsommer.

2. Der Ausaperungsunterbruch durch Schneefall am 18./19. August.

3. Der frühzeitige Ausaperungsabbruch anfangs September.

Die im Sommer 1972 oft schlechte Witterung ermöglichte die Erfassung von 6 Ausaperungsphasen. Figur 4 zeigt das Höhersteigen der Altschneelinie bis zum Endstand am 27. 8. 1972. (Am 8.7. lag noch auf dem ganzen Gletscher Schnee.) Tab. 1 liefert zu den entsprechenden Ausaperungsphasen den ausgeaperten Flächenanteil, das Altschneevolumen und die Altschneevolumenänderungen.

Tabelle 1 Griesgletscher: Ausaperung Sommer 1972

\begin{tabular}{rcl}
\hline Datum & $\begin{array}{l}\text { Ausgeaperte Fläche } \\
\text { (Griesgletscher: 6300 027 } \mathrm{m}^{2} \text { ) } \\
\%\end{array}$ & $\begin{array}{l}\text { Altschnee- } \\
\text { volumen } \\
\mathrm{m}^{3} \text { Wasser }\end{array}$ \\
\hline 8.7. & 0.0 & 7584490 \\
20.7. & 0.2 & 6216726 \\
29.7. & 1.5 & 4958597 \\
10.8. & 8.8 & 4052808 \\
18.8. & 20.7 & 3015791 \\
27.8. & 28.8 & 2992850 \\
\hline
\end{tabular}




\begin{tabular}{|c|c|c|}
\hline \multirow{2}{*}{$\begin{array}{l}\text { Ausaperungs- } \\
\text { periode }\end{array}$} & \multicolumn{2}{|c|}{ Altschneevolumenänderung } \\
\hline & $\mathrm{m}^{3}$ Wasser & $\begin{array}{l}\text { mm Wasser pro Tag } \\
\text { und Altschneefläche }\end{array}$ \\
\hline 8.7.-19.7. & -1367764 & -18 \\
\hline 20.7.-28.7. & -1258129 & -22 \\
\hline 29.7.- 9.8. & $\begin{array}{l}-905789 \\
\end{array}$ & -12 \\
\hline 10.8.-18.8. & -1037017 & -23 \\
\hline $\begin{array}{l}\text { 18. 8.-19.8. } \\
\text { (Neuschnee) }\end{array}$ & +252896 & +40 \\
\hline 19.8.-27.8. & - 275837 & -6 \\
\hline
\end{tabular}

Die ursprüngliche Absicht, Ausaperungsphasen, ausgeaperte Fläche und Altschneevolumen vergleichen zu können, wurde durch die geringe Zahl der Ausaperungsphasen, das heißt, den frühzeitigen Abbruch der Ausaperung verunmöglicht.
Hingegen ergab sich 1972 die Gelegenheit, die Auswirkungen eines Sommerneuschneefalls auf die Ausaperung festzuhalten. Tab. 2 zeigt die mittlere Altschneeablation pro Höhenstufe und Ausaperungsperiode, Tab. 3 liefert die dazu gehörenden, mit dem mittleren Gradienten von $-0,65^{\circ} \mathrm{C}$ pro $100 \mathrm{~m}$ Höhenzunahme reduzierten positiven Tagestemperatursummen der VAW-Station Gries. Den Vergleich dieser Ablationswerte mit den positiven Tagestemperatursummen liefert die Regressionsgleichung

$$
\mathrm{s}=69.7+3.1 \mathrm{~T} \text { mit } \mathrm{k}=0.935
$$

worin s: Altschneeablation in mm Wasser

T: Summe der positiven Tagestemperaturen

k: Korrelationskoeffizient

Tabelle 2 Griesgletscher:

Mittlere Altschneeablation pro Höhenstufe und Ausaperungsperiode (Sommer 1972), in $\mathrm{mm}$ Wasser

\begin{tabular}{lcccccccc}
\hline Höhenstufe & unter & $2500-$ & $2600-$ & $2700-$ & $2800-$ & $2900-$ & $3000-$ & über \\
& 2500 & 2600 & 2700 & 2800 & 2900 & 3000 & 3100 & 3100 \\
\hline Periode & & & &. & & & & \\
$8.7 .-19.7$. & 327 & 276 & 255 & 230 & 219 & 187 & 164 & 158 \\
$20.7 .-28.7$. & 245 & 240 & 224 & 199 & 183 & 162 & 142 & 152 \\
$29.7 .-9.8$. & - & 226 & 227 & 244 & 166 & 159 & 139 & 138 \\
$10.8 .-18.8$. & - & - & 140 & 177 & 197 & 197 & 173 & 178 \\
$19.8 .-27.8$. & - & - & 54 & 60 & 36 & 65 & 30 & 30 \\
\hline
\end{tabular}

Tabelle 3 Summe der positiven Tagesmitteltemperaturen pro Höhenstufe und Periode (Sommer 1972), in ${ }^{\circ} \mathrm{C}$. Basis: VAW-Wetterstation Gries, Reduktion: $-0,65^{\circ} \mathrm{C} / 100 \mathrm{~m}$ Höhe.

\begin{tabular}{rllllllll}
\hline Höhenstufe & $\begin{array}{l}\text { unter } \\
2500\end{array}$ & $\begin{array}{l}2500- \\
2600\end{array}$ & $\begin{array}{l}2600- \\
2700\end{array}$ & $\begin{array}{l}2700- \\
2800\end{array}$ & $\begin{array}{l}2800- \\
2900\end{array}$ & 3000 & 3100 & \multicolumn{2}{l}{$\begin{array}{l}2900- \\
3100\end{array}$} \\
\hline Periode & & & & & & & & \\
8.7.-19.7. & 71.9 & 64.5 & 58.4 & 51.7 & 48.0 & 40.0 & 34.5 & 29.1 \\
$20.7 .-28.7$. & 62.5 & 56.2 & 50.8 & 44.5 & 39.1 & 32.8 & 27.4 & 21.9 \\
$29.7 .-9.8$. & 64.7 & 57.0 & 50.4 & 42.8 & 36.8 & 29.8 & 23.9 & 18.6 \\
$10.8 .-18.8$. & 65.0 & 59.4 & 54.6 & 49.0 & 44.2 & 38.6 & 33.8 & 29.2 \\
$19.8 .-27.8$. & 25.6 & 20.8 & 17.2 & 13.0 & 10.2 & 8.1 & 6.4 & 5.0 \\
\hline
\end{tabular}


Fig. 1: Grimsel (MZA), 1970 mü. M.

Mittlere Tagestemperaturen im Sommer 1972

Fig. 2: Grimsel (MZA), 1970 mü. M.

Schneehöhen im Sommer 1972

Fig. 3: Lago Vannino (V. Antigorio), 2183 mü. M.

Niederschläge im Sommer 1972

Fig. 4: Griesgletscher: Ausaperungsphasen im Sommer 1972

1

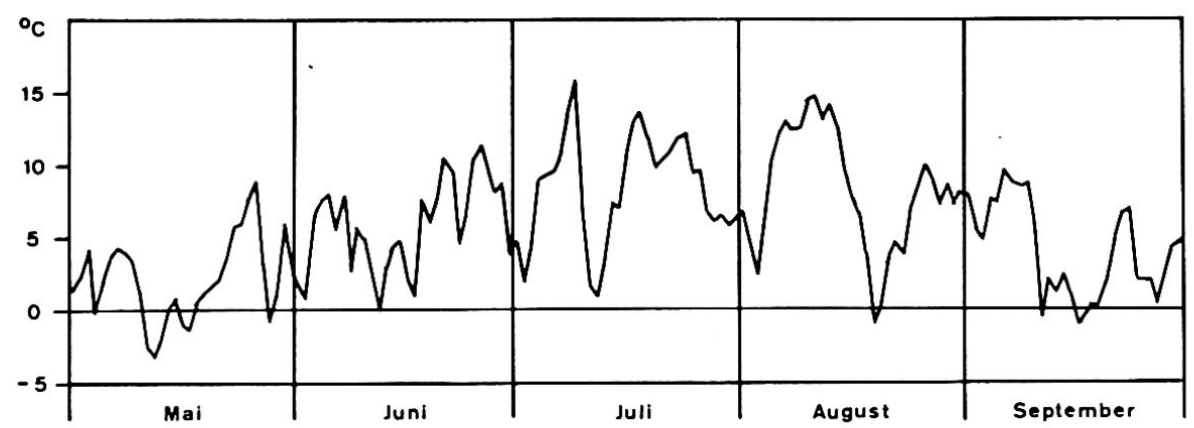

2

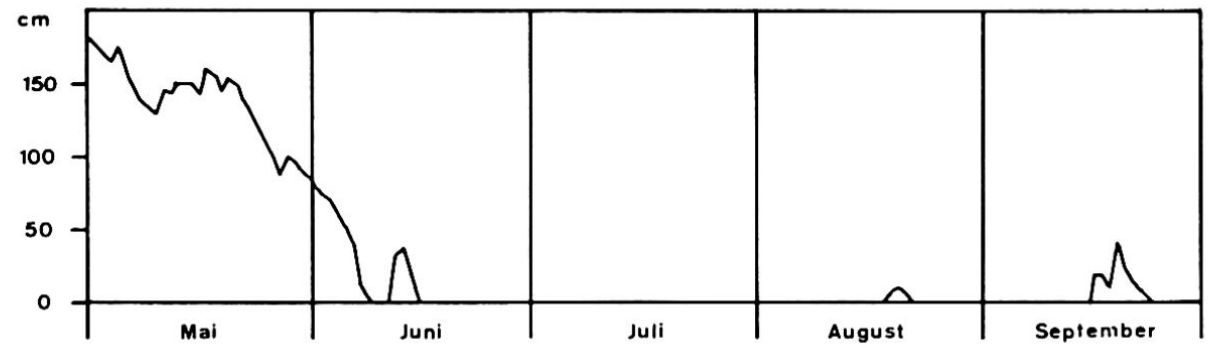

3
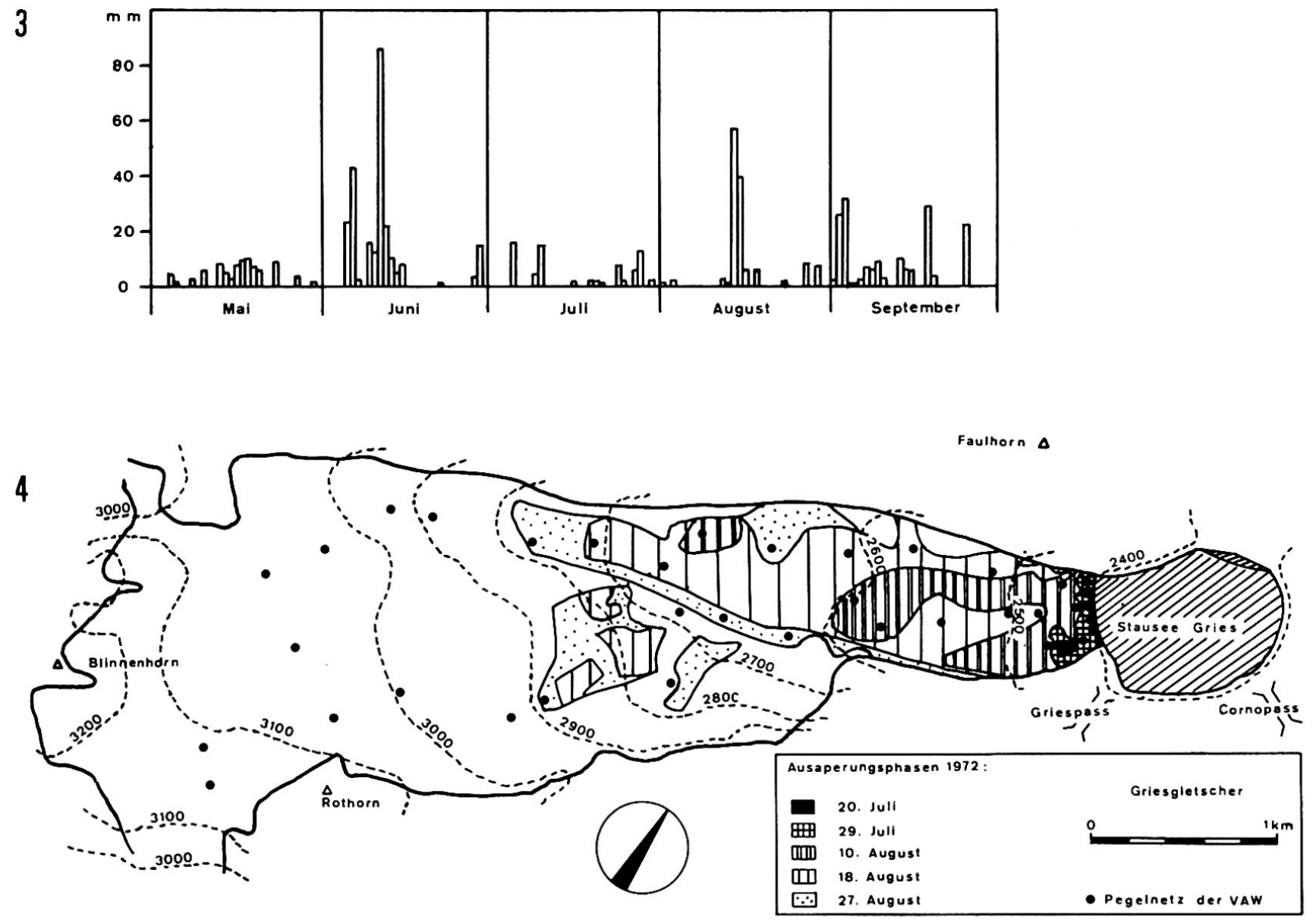
Fig. 5: Griesgletscher im Sommer 1972: Altschneeablation und Summe der positiven Tagestemperaturen (vgl. Tabellen 2 und 3)
Fig. 6: Griesgletscher im Sommer 1972: Altschneeablation (vgl. Tabelle 1)

Fig. 7: Massenhaushalt des Griesgletschers und Klimaverlauf auf dem Gütsch ob Andermatt (2288 mü. M.), 1962-1972 (vgl. Tabelle 4)

Fig. 8: Griesgletscher: Korrelation des Massenhaushaltes mit den Sommerniederschlagsreihen umliegender Stationen, 1962-1972 (vgl. Tabelle 6).

\section{5}

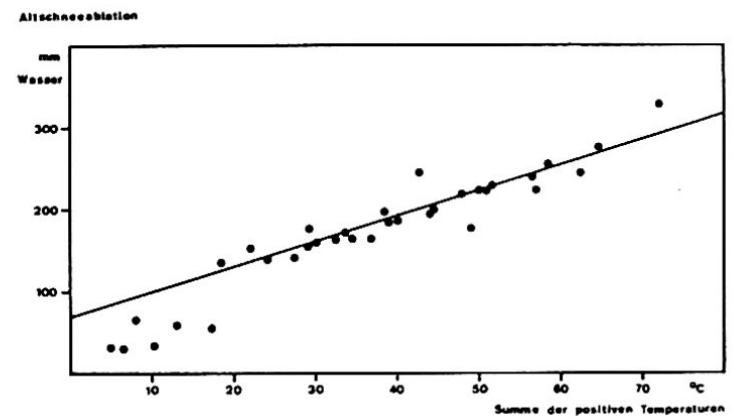

6

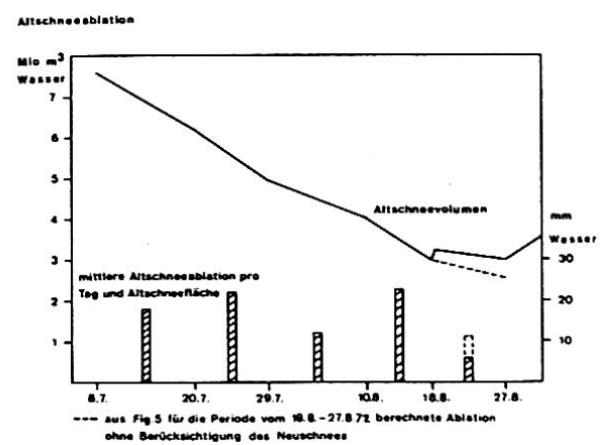

7
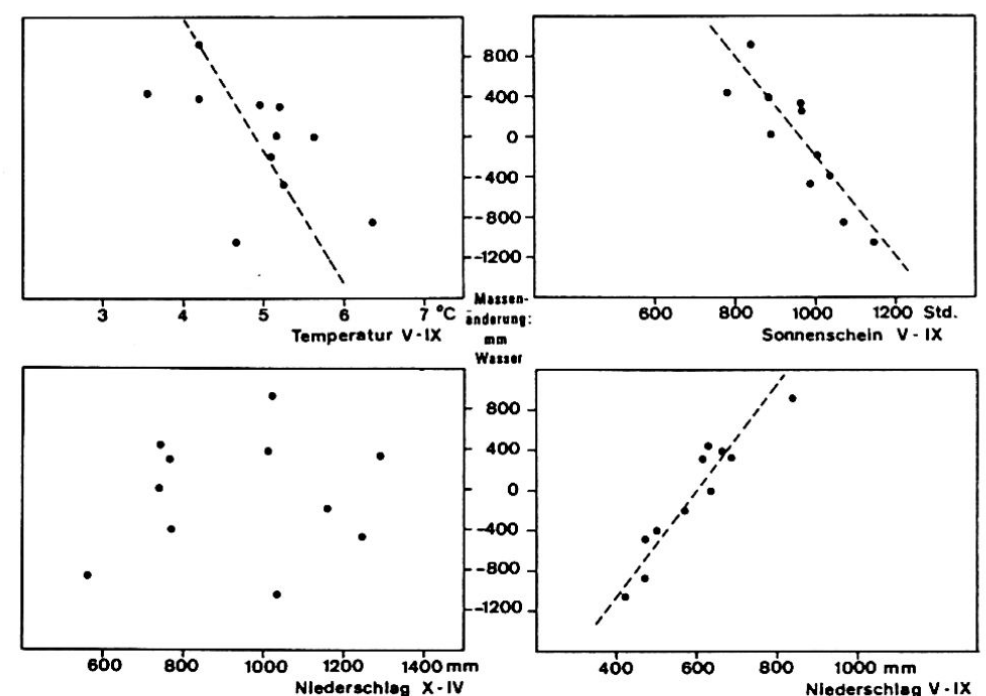

8

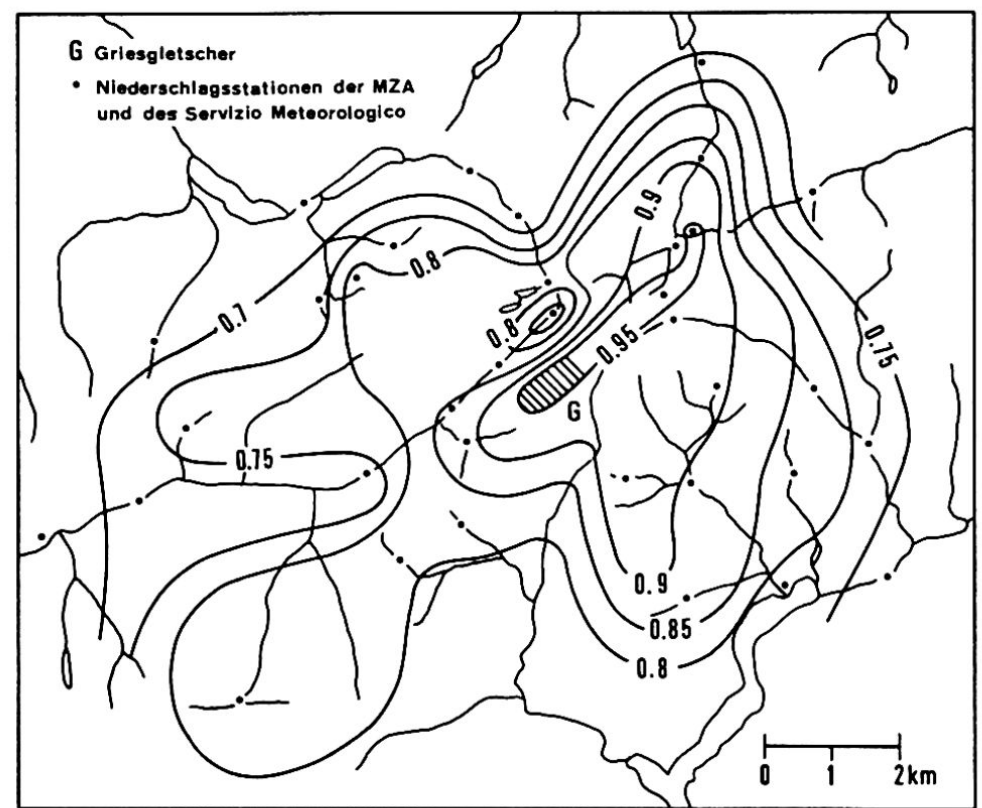


Wird nun mit Hilfe dieser Regressionsgleichung die Altschneeablation der Periode 18.8.-27.8. 1972 berechnet, erhält man einen Volumenverlust von $425759 \mathrm{~m}^{3}$ Wasser. Dem steht ein tatsächlicher Verlust von nur $275837 \mathrm{~m}^{3}$ Wasser gegenüber (Fig. 6).

Der Sommerneuschneefall vom 18./19.8. 1972 vermag diese Differenz zu erklären: Durch das höhere Strahlungsreflektionsvermögen (höherer Albedowert) des Neuschnees wird der Ausaperungsproze $B$ entscheidend beeinflußt. Nicht nur die Altschneedecke wird dabei isoliert, auch Firn- und Eisablation werden je nach Neuschneemächtigkeit und Strahlung für eine gewisse Zeit stillgelegt.

\section{Massenhaushalt und Klimaverlauf}

Im Auftrag der Aegina-Kraftwerke AG ermittelt die VAW seit 1962 anfangs Oktober die Massenbilanz des
Griesgletschers. Die vorliegende 1ljährige Massenhaushaltsreihe (Tab. 4) erlaubt es nun mit Hilfe von Korrelationsrechnungen, den Einfluß einzelner Klimaelemente auf Massenveränderungen des Gletschers abzuklären. Beigezogen wurde die zum synoptischen Netz der MZA gehörende Station Gütsch ob Andermatt $(2288 \mathrm{~m})$. In Übereinstimmung mit dem jährlichen Bericht «Die Gletscher der Schweizer Alpen» der VAW (Prof. Kasser) werden dem Sommer die Monate Mai bis September, dem Winter Oktober bis April zugeordnet. Als Klimaelemente wurden gewählt:

- Sommertemperatur

- Sommerniederschlag

- Sommersonnenscheindauer

- Winterniederschlag

Tab. 4 liefert das Datenmaterial, Figur 7 zeigt den Vergleich zwischen den Massenänderungen des Gletschers und den Klimaelementen.

Tabelle 4 Massenhaushalt Griesgletscher und Klimaverlauf Gütsch ob Andermatt (2288 m), 1962-1972

\begin{tabular}{|c|c|c|c|c|c|}
\hline Jahr & $\begin{array}{l}\text { 1) } \\
\text { Massenbilanz } \\
\text { mm Wasser }\end{array}$ & $\begin{array}{l}\text { 2) } \\
\text { Sonnenschein } \\
\text { Mai-Sept. } \\
\text { Std. }\end{array}$ & $\begin{array}{l}\text { 3) } \\
\text { Temperatur } \\
\text { Mai-Sept. } \\
{ }^{\circ} \mathrm{C}\end{array}$ & $\begin{array}{l}\text { 2) } \\
\text { Niederschlag } \\
\text { Mai-Sept. } \\
\text { mm }\end{array}$ & $\begin{array}{l}\text { 2) } \\
\text { Niederschlag } \\
\text { Okt.-April } \\
\text { mm }\end{array}$ \\
\hline $\begin{array}{l}1962 \\
1963 \\
1964 \\
1965 \\
1966 \\
1967 \\
1968 \\
1969 \\
1970 \\
1971 \\
1972 \\
\end{array}$ & $\begin{array}{r}-1068 \\
31 \\
-\quad 862 \\
925 \\
-\quad 202 \\
328 \\
381 \\
294 \\
-\quad 484 \\
-\quad 409 \\
444\end{array}$ & $\begin{array}{r}1146 \\
891 \\
1070 \\
840 \\
1005 \\
965 \\
886 \\
961 \\
984 \\
1034 \\
781\end{array}$ & $\begin{array}{l}4.66 \\
5.14 \\
6.34 \\
4.12 \\
5.10 \\
4.94 \\
4.20 \\
5.20 \\
5.26 \\
5.62 \\
3.54\end{array}$ & $\begin{array}{l}422 \\
634 \\
469 \\
837 \\
571 \\
685 \\
663 \\
613 \\
472 \\
499 \\
627\end{array}$ & $\begin{array}{r}1036 \\
738 \\
561 \\
1020 \\
1158 \\
1287 \\
1009 \\
764 \\
1242 \\
772 \\
743\end{array}$ \\
\hline \multicolumn{2}{|c|}{ Korrelationskoeffizient } & -0.8785 & -0.6304 & 0.9506 & 0.1187 \\
\hline
\end{tabular}

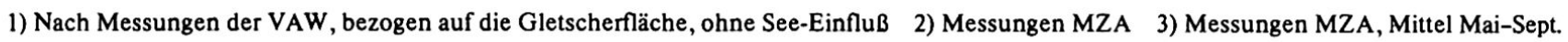


Erstaunlich gute Ergebnisse liefern Sommerniederschlag und Sommersonnenscheindauer. Beide Elemente stehen in engem Zusammenhang mit der Strahlung und bestätigen damit die Bedeutung der sommerlichen Strahlung für die Ablation. Deutlich unter diesen beiden Werten liegt der Einfluß der mittleren Sommertemperatur. Verhältnismäßig gering scheint der Einfluß des Winterniederschlages zu sein. Es ist jedoch zu berücksichtigen, daß das Winterhalbjahr am 30. 4. abgeschlossen wird. Entscheidende Schneemengen fallen in höheren Lagen jedoch oft im Mai und Juni (vgl. Winterberichte des Instituts für Schneeund Lawinenforschung Weißfluhjoch-Davcs). Diese Schneemengen führen zum Aufstocken der Schneedecke und zur Verzögerung der Ausaperung. Da diese Niederschlagsmengen bereits dem Sommer zugezählt werden, verstärken sie zusätzlich den Einfluß des Sommerniederschlags auf die Massenbilanz des Gletschers.

\section{Die Lage des Griesgletschers im alpinen Klimaraum}

Die oben erwähnten Untersuchungen wurden für die gut korrelierenden Elemente Sommerniederschlag und Sommersonnenscheindauer auf weitere umliegende Stationen ausgedehnt, um damit die klimatische Wirksamkeit der umliegenden Regionen auf den Gletscher abzuklären.

Tabelle 5 Korrelationskoeffizienten (K): Massenhaushalt Griesgletscher - Sonnenscheindauer Mai-Sept. umliegender Stationen, berechnet aus den Werten der Jahre 1962-1972

\begin{tabular}{lrl}
\hline Station & Höhe & K \\
\hline Sion & 551 & -0.9371 \\
Zermatt & 1632 & -0.8905 \\
Gütsch & 2288 & -0.8785 \\
Jungfraujoch & 3576 & -0.8426 \\
\hline
\end{tabular}

Tab. 5 zeigt die Korrelationskoeffizienten für die Sommersonnenscheindauer. Hier liefert die Talstation Sion das beste Ergebnis, während mit zunehmender Höhe die Koeffizienten abnehmen, obwohl der Griesgletscher zwischen 2400 und $3300 \mathrm{~m}$ Höhe liegt. Die Erklärung dieses Ergebnisses dürfte in der häufigeren Nebelbildung durch sommerliche Cumulusbewölkung in Kamm- und Gipfellagen zu suchen sein. Diese Bewölkung vermag größere Flächen wie den Griesgletscher jedoch kaum von der Sonnenstrahlung abzuschirmen, ergibt jedoch bei höher gelegenen Stationen geringere Sonnenscheinwerte. Das unterschiedliche Auftreten dieser Bewölkung vermindert den Korrelationskoeffizienten.

Tabelle 6 Korrelationskoeffizienten (K): Massenhaushalt Griesgletscher - Niederschlag Mai-Sept. umliegender Stationen, berechnet aus den Werten der Jahre 1962-1972

\begin{tabular}{ll}
\hline Station & $\mathrm{K}$ \\
\hline St. Gotthard & 0.9551 \\
Gütsch & 0.9506 \\
Andermatt & 0.9399 \\
Gurtnellen & 0.8999 \\
Altdorf & 0.7029 \\
Airolo & 0.9111 \\
Fusio & 0.9048 \\
Bosco-Gurin & 0.9020 \\
Cevio & 0.8946 \\
Camedo & 0.8938 \\
Lavorgo & 0.8482 \\
Lago d'Avino & 0.8306 \\
Frasco & 0.8079 \\
Simplon & 0.8076 \\
Biasca & 0.7959 \\
Locarno & 0.7806 \\
Bellinzona & 0.7121 \\
Binn & 0.8992 \\
Fiesch & 0.8989 \\
Reckingen & 0.8457 \\
Zermatt & 0.8072 \\
Leukerbad & 0.7744 \\
Brig & 0.7400 \\
Oberwald & 0.7262
\end{tabular}




\begin{tabular}{ll}
\hline Station & $\mathrm{K}$ \\
\hline Chippis & 0.7132 \\
Sion & 0.6137 \\
Grimsel & 0.8469 \\
Kl. Scheidegg & 0.8036 \\
Grindelwald & 0,7819 \\
Mürren & 0.7071 \\
Adelboden & 0.6978 \\
Guttannen & 0.6694 \\
Interlaken & 0.6184 \\
Meiringen & 0.6031 \\
Disentis & 0,6958 \\
Ilanz & 0.6140 \\
\hline
\end{tabular}

Das relativ dichte Netz der Niederschlagsmeß-Stationen ermöglichte das Aufzeichnen der Werte in einer Korrelatenkarte (Tab. 6, Figur 8). Sie zeigt eine deutliche Zugehörigkeit des Griesgletschers zum Niederschlagsregime der Zentralalpen. Hohe Korrelationskoeffizienten im Tessin und oberen Reußtal bestätigen diese Aussage. In den Berner und südlichen Walliser Alpen sind die Koeffizienten von der Höhe abhängig. Das Sommerniederschlagsgeschehen tieferer Stationen wird häufig durch lokale Gewitter geprägt. Die Niederschlagsreihen dieser Stationen weisen deshalb einen geringeren Einfluß auf. Auffallend ist zudem die geringe Korrelation naher Stationen im Oberwallis (z. B. Oberwald).

Damit sind einige zentrale Fragen der Alpenklimatologie angeschnitten, so zum Beispiel:

- die Charakterisierung typischer alpiner Klimaregionen, z. B. unter Berücksichtigung einzelner Wetterlagen

- die Abgrenzung und Überlagerung dieser Regionen in den Zentralalpen

- der Einfluß alpiner Quertalungen (Wallis-UrserenVorderrhein) auf die Wetterwirksamkeit einzelner Wetterlagen.

\section{Zusammenfassung}

Relief, Exposition und Höhe über Meer bestimmen die Schneehöhe und die Abschmelzenergie und damit die Ausaperung. Durch die Konstanz der 3 Faktoren ergibt sich jedes Jahr ein gleich ablaufendes Ausaperungsbild. Unterschiedliche klimatische Verhältnisse bewirken den unterschiedlichen Zeitpunkt des Eintreffens einzelner Ausaperungsbilder.

Die Untersuchung der Ausaperung und des Klimas im Sommer 1972 auf dem Griesgletscher (Oberwallis) zeigte den großen Einfluß sommerlicher Neuschneefälle auf die Ablation.

Der Vergleich der 11jährigen Massenhaushaltsreihe mit Klimaelementen der Station Gütsch ob Andermatt $(2288 \mathrm{~m})$ lieferte gute Ergebnisse für Sommerniederschlag und Sommersonnenscheindauer. Diese beiden Elemente bestätigen den für die Ablation entscheidenden Einfluß der Strahlung.

Mit der Ausdehnung der Korrelationsrechnungen auf weitere Stationen konnte die klimatische Zugehörigkeit des Griesgletschers zum Zentralalpenraum nachgewiesen werden.

PS: Es handelt sich bei diesem Beitrag um einen Auszug aus einer Diplomarbeit, welche am Geographischen Institut der Universität Bern, Abt. Prof. Dr. B. Messerli, unter Mitwirkung der Abteilung für Hydrologie und Glaziologie (Prof. Dr. Kasser) der Versuchsanstalt für Wasserbau, Hydrologie und Glaziologie der ETHZ ausgeführt wurde.

\section{Literatur}

AMBACH W., EISNER H. (1967) Klimatologische Interpretation eines Firnpollenprofils.

9. Internat. Tagung für alpine Meteorologie in Brig und Zermatt, 1966, Veröffentlichungen der MZA Nr. 4

FLIRI F. (1964): Zur Witterungsklimatologie sommerlicher Schneefälle in den Alpen.

Wetter und Leben, Jg. 16

FLIRI F. (1969): Statistik und Diagramm.

Das geographische Seminar, Westermann, Braunschweig.

FÖHN P. (1971): Methoden der Massenbilanzmessung bei großen Schneehöhen, untersucht im Firngebiet des Großen Aletschgletschers. Diss., Zürich.

FRIEDEL H. Gesetze der Niederschlagsverteilung im Hochgebirge. Wetter und Leben, Jg. 4 
HOECK E. (1952): Der Einfluß der Strahlung und der Temperatur auf den Schmelzprozeß der Schneedecke. Beiträge zur Geologie der Schweiz - Geotechnische Serie - Hydrologie, Lieferung 8.

HOINKES H. (1967): Gletscherschwankungen und Wetter in den Alpen. Veröffentlichungen der MZA Nr. 4.

HOINKES H., WENDLER G. (1968): Der Anteil der Strahlung an der Ablation von Hintereis- und Kesselwandferner (Ötztaler Alpen) im Sommer 1958.

Archiv für Meteorologie, Geophysik und Bioklimatologie, Serie B, Jg. 16.

International Commission of Snow and Ice of the International Association of Scientific Hydrology (1969). Mass - Balance Terms. Journal of Glaciology, Vol. 8. KUHN W. (1950): Zusammenhang zwischen Firnzuwachs, Sommertemperatur und Niederschlag nach Messungen auf dem Clariden- und Silvrettagletscher. KUHN w. (1953): Statistische Ergebnisse von Firnzuwachsmessungen. Verhandlungen der Schweiz. Naturforschenden Gesellschaft.
SLUPETZKY H. (1971): Der Verlauf der Ausaperung am Stubacher Sonnenbtickkees (Hohe Tauern).

Mitt. der Österr. Geogr. Ges., Jg. 113.

Versuchsanstalt für Wasserbau, Hydrologie und Glaziologie an der ETH Zürich (Prof. Kasser): Die Gletscher der Schweizer Alpen. Publikation der Gletscherkommission der Schweiz. Naturforsch. Gesellschaft. ZINGG TH. (1948/49): Beitrag zur Kenntnis des Schmelzwasserabflusses der Schneedecke. Schnee und Lawinen, Winterberichte des Eidg. Inst. für Schnee- und Lawinenforschung Davos

Datenmaterial: Schweiz. Meteorologische Zentralanstalt (MZA): Annalen; Witterungsbericht.

Eidg. Inst. für Schnee- und Lawinenforschung: Schnee und Lawinen (Winterberichte)

Versuchsanstalt für Wasserbau, Hydrologie und Glaziologie: Untersuchungsberichte Griesgletscher (unveröff.).

Aeronautica Militare ITAV - Servizio Meteorologico: Niederschlagsdaten Lago Vannino und Lago d'Avino (z. T. unveröff.). 